\title{
Ereignisinterne Adjunkte in einem typologisch orientierten Sprachvergleich Deutsch-Türkisch
}

der verehrten Kollegin Brigitte Handwerker zum 60. Geburtstag

\begin{abstract}
Der nachfolgende Beitrag untersucht Form-Funktionsbeziehungen in einem spezifischen Bereich adverbialer Modifikation im Deutschen und Türkischen, nämlich bei den ereignisinternen Adjunkten. Abschnitt 1 entwickelt - mit übereinzelsprachlichem Gültigkeitsanspruch - die Begrifflichkeiten, anhand derer die Untersuchung vorgenommen wird. Abschnitt 2 wendet diese auf das Deutsche und das Türkische an. Abschnitt 3 formuliert ein kontrastives Zwischenergebnis, das in Abschnitt 4 mittels des Versuchs der Identifikation typologischer Korrelate der festgestellten Unterschiede zu einer typologischen Hypothese erweitert wird. ${ }^{1}$

Der Beitrag ist dem holistischen sprachtypologischen Programm verpflichtet, das die Systematiken aufzeigen will, die den identifizierten sprachlichen Strukturen unterliegen und das diese Systematiken als Instanzen allgemeinerer Prinzipien der Variation und Übereinstimmung von sprachlichen Systemen darstellen will. Es wird beschreibend vorgegangen; aus der Beschreibung soll ein Verständnis des Gegenstands erzielt werden. ${ }^{2}$ Als deskriptives Werkzeug dienen die Begrifflichkeiten der funktionalen Typologie und der Semantik.

Die Auseinandersetzung mit dem Untersuchungsgegenstand ,ereignisinterne Adjunkte" geschieht in dem für typologische Untersuchungen höchst engen Rahmen der Untersuchung von nur zwei Sprachen. Dies hat Vorteile, die sich insbesondere auf die angelegte Breite und den primär semantischen Ausgangspunkt der Untersuchung beziehen. Es hat gleichzeitig Nachteile, die sich vor allem auf die Verallgemeinerbarkeit oder implikative Kraft der identifizierten Zusammenhänge beziehen. ${ }^{3}$ Die Vorteile gilt es zu nutzen, die Nachteile zu beachten.
\end{abstract}

\section{Begrifflichkeiten, Herangehensweise}

Bevor ich zu dem spezifischen Thema der ereignisinternen Adjunkte im Deutschen und Türkischen komme, muss zunächst eine Reihe von Begrifflichkeiten abgearbeitet werden. Sie etablieren das Tertium Comparationis, in dessen Rahmen die einzelsprachlichen Beschreibungen in Abschnitt 2 plat-

1 Der Beitrag entwickelt Gedanken aus meiner Habilitationsschrift (Schroeder 2004) und aus Schroeder (2008) weiter.

2 „Sprachwissenschaft ist ein deskriptives Geschäft, bei dem über die Beschreibung allerdings auch eine Erklärung oder genauer gesagt ein Verständnis des Gegenstandes erzielt werden soll“" (Maas 2000, S. 2).

3 Siehe hierzu Ekkehard Königs Beitrag in diesem Band. 
ziert werden. Die Begrifflichkeiten betreffen das hier vorausgesetzte Verständnis von „Adjunkten“ und ihre syntaktische und semantische Klassifizierung (1.1) sowie die Frage nach der „,semantischen Orientierung“ bei den hier behandelten ereignisinternen Adjunkten (1.2). ${ }^{4}$

\subsection{Adjunkte - syntaktisch und semantisch}

Unter „Adjunkt“ ist hier ein nichtobligatorischer Satzteil verstanden, der modifizierendes Element irgendeiner Phrase ist. Der allgemeine Terminus „Adjunkt" wird hier den Termini „Adverb“, das eine Wortart bezeichnet, und „Adverbial“, das in linguistischen Beschreibungen mal synonym für Adjunkte des Satzes eingesetzt wird, mal ein Adjunkt mit einer spezifischen modifikatorischen Kraft meint, und auch auf obligatorische Formen mit Komplementstatus (die „Adverbialkomplemente“ der IDS-Grammatik, vgl. Zifonun/Hoffmann/Strecker 1997, S. 1099 ff.) angewandt wird, vorgezogen.

Adjunkte lassen sich syntaktisch nach dem Phrasentyp klassifizieren, zu dem sie gehören. Im Zusammenhang dieser Untersuchung spielen eine Rolle:

- die voll gesättigte Verbalphrase, ${ }^{5}$ also das Prädikat mit seinen Argumenten (einschließlich des Subjekts) und den auf dieser Ebene wirksamen Adjunkten,

- das Prädikat selbst (als Teil der Verbalphrase), das gemeinsam mit seinen Adjunkten ein „komplexes Prädikat“ bilden kann und

- die Nominalphrase, in der das Kernnomen voran- oder nachgestellte modifizierende Adjunkte zu sich nehmen kann.

Im Folgenden konzentriere ich mich auf nicht-satzwertige Adjunkte in der Verbalphrase. „Nicht-satzwertig“ bedeutet hier, dass diese Adjunkte die Bedingungen erfüllen, dass sie i) nominale Phrasen, Adpositionalphrasen oder nichtfinite verbale Phrasen sind und ii) keine eigenständigen satzprosodischen Einheiten bilden.

$4 \quad$ Hier verwendete Beispiele aus dem Deutschen stammen, solange sie nicht trivial sind bzw. solange nicht anders angegeben, aus dem COSMAS-Korpus des Instituts für Deutsche Sprache (IDS). Ich habe mir jedoch erlaubt, dem Korpus entnommene Sätze teilweise zu kürzen. Beispiele aus dem Türkischen stammen, solange nicht anders angegeben, aus dem „Turkish Text Corpus“ (TTC), das von Gerjan van Schaaik (Leiden) zusammengestellt wurde (van Schaaik (Hg.) 1990-2000). Beide Korpora enthalten wissenschaftliche, belletristische und journalistische Prosa, die aus dem Vorrat des modernen schriftsprachlichen Standards der beiden untersuchten Sprachen stammt.

5 Bei Foley/Van Valin (1984) und Van Valin/LaPolla (1997) wird für diese Ebene die Bezeichnung „core“ verwendet; Lehmann (1988) verwendet „verb phrase“; bei Zifonun/Hoffmann/ Strecker (1997) wird von „Verbgruppe (V0)“ gesprochen; ich verwende „Verbalphrase“. 
Im Rahmen dieser formalen Klassifikation interessieren uns im Zuge einer semantischen Klassifikation von Adjunkten ${ }^{6}$ solche, die Elemente des Ereignisses selbst modifizieren oder Elemente des Ereignisses selbst sein können. In Anlehnung an Frey/Pittner (1998) und Pittner (1999, 2002) nenne ich diese Adjunkte „ereignisinterne Adjunkte“.

Auch hier können wir mehrere Untergruppen bilden:

- Komitative und Instrumentale (... ging mit ihr die Straße entlang; ... schlug mit dem Hammer auf den Nagel) führen weitere umstandsbezogene Referenten in das Ereignis ein - entweder stärker referentenorientiert, indem sie als Komitative die gemeinsame Vorgangsausführung mit einem weiteren Partizipanten ausdrücken, oder stärker vorgangsorientiert, indem sie als Instrumentale das Instrument zur Vorgangsausführung angeben.

- Adverbiale der Intensität („,measure adverbs“ in Tenny 2000, z.B. ... schloss die Tür halb; ... fiel fast die Treppe hinunter) modifizieren den Grad der Ausführung des Vorgangs oder der Aktion.

- Distributive (... spazierten gruppenweise durchs Dorf; ... teilte die Handouts paarweise aus) beinhalten eine repetitive aktionale Modifikation des Vorgangs bzw. der Aktion und quantifizieren gleichzeitig einen der beteiligten Referenten.

- Adverbiale der Art und Weise (... schloss heftig die Tür, [die Tür] fiellaut ins Scbloss) drücken die Art und Weise aus, in der ein Vorgang oder eine Aktion geschieht bzw. ausgeführt wird.

- Depiktive sekundäre Prädikate („Depiktive“) (... trank den Kaffee eiskalt, ... stand in Hut und Mantel in der Tür) drücken einen Zustand eines der am Ereignis beteiligten Referenten („Bezugsreferent") während des Vorgangs-/Aktionsablaufs aus.

Ich konzentriere mich nun auf zwei dieser Gruppen, nämlich auf depiktive sekundäre Prädikate, im Folgenden nach Geuder $(2000,2004)$ und SchultzeBerndt/Himmelmann (2004) „Depiktive“ genannt, und auf Adverbiale der Art und Weise. Dabei schließe ich nicht aus, dass Schlussfolgerungen, die sich aus der kontrastiven Analyse dieser beiden Gruppen ergeben, sich auch auf die anderen Gruppen erweitern lassen. Dies zu konkretisieren kann hier jedoch nicht geleistet werden.

Ich beziehe mich hier auf typologisch verwertbare Klassifikationen wie Dik et al. (1990), Ramat/Ricca (1998), ergänzt um Diskussionen bei Frey/Pittner (1998), Pittner (1999, 2002), Bellert (1977) und Maienborn/Schäfer (erscheint demnächst). 
1.2 Semantische Orientierung bei Depiktiven und Adverbialen der Art und Weise

Es gibt eine umfangreiche Literatur, in der die Depiktive vor allem aufgrund ihrer spezifischen nominal- und eben nicht verbbezogenen modifikatorischen Kraft, z.T. auch in ihrem syntaktischen Status und in ihrer syntaktischen Klassifikation mehr oder weniger radikal von den Adverbialen der Art und Weise unterschieden werden. ${ }^{7}$ Ich sehe hierzu aufgrund der syntaktischen (beide sind Adjunkte der Verbalphrase) und semantischen (beide sind ereignisinterne Adjunkte) Parallelen keinen Anlass und verweise auf Zifonun/Hoffmann/Strecker (1997, S. 1189 ff.), wo mit dem Terminus „komplementbezogene Verbgruppenadverbiale“ die Adverbialität der Depiktive betont wird. So soll in diesem Abschnitt der Versuch unternommen werden, Depiktive und Adverbiale der Art und Weise als eine Gruppe von ereignisinternen Adjunkten zusammen zu fassen, die im nächsten Schritt auf der Grundlage ihrer semantischen Orientierung innerhalb des Ereignisses subklassifiziert werden, d.h. auf der Grundlage des Beitrags, den sie zur Konstitution des Ereignisses leisten. Dabei folge ich - mit einigen Erweiterungen und Veränderungen - den wichtigen diesbezüglichen Beiträgen von Platt/Platt (1972), Bartsch (1976, S. 152 f.), Buysschaert (1982, S. 150 f.) und Geuder $(2000,2004)$ und unterscheide zwischen i) referentenorientierten, ii) aktivitätsorientierten, iii) vorgangsorientierten, iv) wirkungsorientierten und v) umstandsorientierten ereignisinternen Adjunkten. ${ }^{8}$

\subsubsection{Referentenorientierte ereignisinterne Adjunkte}

Unter die referentenorientierten ereignisinternen Adjunkte fasse ich die Depiktive. Wie oben bereits festgelegt, drücken sie einen Zustand eines der

Größtenteils wird die Nähe dieser Konstruktionen zu Prädikaten betont: ,sekundäre Prädikate“ („,secondary predicates“) (Nichols 1978; Napoli 1989; Aarts 1995; Müller 2003), „Koprädikative“ (Plank 1985; Müller-Bardey 1990), ,freie Prädikative“ (Eisenberg 1999, S. 224), „Prädikativum“ (Pinkster 1990), „Prädikative“ (Abraham 1995; Pittner 1999). Andere Termini betonen die nominale Orientierung der Depiktive, also ihren Zusammenhang mit den Attributen: „prädikative Attribute“ (Helbig/Buscha 1989, S. 493; Bausewein 1990), ,adverbial attributes“ (Bartsch 1976), ,prädikative Adjektive“ (Renz 1993, S. 29), „, supplementive adjective clauses“ (Quirk et al. 1985, S. 427), „temporally restricted adjectives“ bzw. „temporalisierte Adjektive“ (Dowty 1972; Rath 1972; Nikula 1982). Auch die Bezeichnung „Adjunkt“ bei Engel (1988, S. 628) bezieht sich auf die nominale Orientierung: Engel subsumiert damit Depiktive unter die Gruppe der dislozierten Elemente der Nominalphrase. Ein wieder anderer Terminus, „Kurzsätze“/,Sätzchen“ (,small clauses“) (Chomsky 1981, S. 169; Stowell 1983; Aarts 1992; Steube 1994; Staudinger 1997, siehe auch Fabricius-Hansen/Ramm in diesem Band), stellt die Depiktive in die Nähe von Sätzen.

$8 \quad$ Kurze Diskussionen auch bei Pittner (1999, S. 95 f.), Dik et al. (1990), Jackendorff (1972, S. 58), Quirk et al. (1985, S. 559 ff.) und Renz (1993, S. 29 f.). Eine alternative Klassifikation, die stärker das pragmatische Verhältnis zwischen dem Adverbial der Art und Weise und dem Ereignis beachtet, findet sich bei Zifonun/Hoffmann/Strecker (1997, S. 1199 ff.). 
am Ereignis beteiligten Referenten („Bezugsreferent ${ }^{\circ}$ ) während des Ereignisablaufes aus. Es ist eine Paraphrase möglich, bei der das Hauptprädikat ohne das referentenorientierte Adjunkt einen vollständigen Satz bildet und das referentenorientierte Adjunkt in einem Folgesatz ein Prädikat über denjenigen Referenten des Vorgängersatzes bildet, über den es als Adjunkt etwas prädiziert. Der Folgesatz enthält ein Temporaladverb („dabei“, „währendessen“ o.Ä.), das sicherstellt, dass die Zustandsprädikation in die Zeit der im Vorgängersatz ausgedrückten Ereignisprädikation fällt. Zu erwarten ist, dass die Paraphrase die prädikative Beziehung zwischen dem referentenorientierten ereignisinternen Adjunkt und dem Bezugsnominal eindeutig und vollständig wiedergibt:

(1) Er geht vergnügt nach Hause.

$\rightarrow$ Er geht nach Hause und ist (dabei/währenddessen) vergnügt.

Talim-den hep sirlsılklam bir bal-de
Übung-ABL immer klitschnass ein Zustand-LOK
dön-dü-k.
zurückkehren-PRÄT-1PL
„Wir kehrten immer klitschnass von der Übung zurück.“
$\rightarrow$ Dön-dü-k $\quad$ ve bu arada hep sirllsılam-di-k.
zurückkehren-PRÄT-1PL und dabei immer klitschnass-PRÄT-1PL
$\quad$ „Wir kehrten zurück und waren dabei immer klitschnass.“

Mit Elementen, die keine referentenorientierten ereignisinternen Adjunkte sind, ist die Paraphrase entweder unangemessen, wie bei dem resultativen sekundären Prädikat in (3),${ }^{10}$ oder die Paraphrase gibt die prädikative Beziehung im Ausgangssatz nicht vollständig und eindeutig wieder, wie bei schnell in (4), wo die Paraphrase mit der substantivierten Handlung als Subjekt die Vorgangsorientierung vollständiger ausdrückt als die referentenorientierte Paraphrase:

9 Der Paraphrasetest hat natürlich seine Tücken, nicht zuletzt in einer kontrastiven Arbeit: Ein/e anonyme/r Gutachter/in weist mich auf Schwierigkeiten mit der Paraphrase hin, z.B. bei den referentenorientierten Adverbien der Körperhaltung, die weder im Deutschen noch im Türkischen als Prädikative zu Kopulaverben einsetzbar sind, vgl. Er flog kopfüber die Treppe binunter. $\rightarrow{ }^{*}$ Er flog die Treppe hinunter und war dabei kopfüber. Die Paraphrase wird in dieser Arbeit als Instrument zur Identifikation einer semantischen Beziehung verstanden und das bedeutet, dass grammatisch problematische Paraphrasen daraufhin überprüft werden müssen, ob die grammatische Problematik mit der sprachenspezifischen Prädikatsfähigkeit und -unfähigkeit von bestimmten Adjunkten zusammenhängt oder mit dem Nichtvorhandensein der zu identifizierenden semantischen Beziehung. Ist Ersteres der Fall, gilt die Paraphrase auch dann, wenn sie grammatisch problematisch ist (so auch Buysschaert 1982, S. 91-93).

10 Resultative sekundäre Prädikate sind syntaktisch im Übrigen nicht auf der Ebene der Verbalphrase, sondern auf der Ebene des Prädikats zu verorten, mit dem zusammen sie ein ,komplexes Prädikat" bilden (siehe auch Schultze-Berndt/Himmelmann 2004, S. 65-67 und Pittner 1999, S. 97). 
(3) Er hämmerte das Blech flach.

$* \rightarrow$ Er hämmerte das Blech und es war dabei flach.

(4) Er geht schnell nach Hause.

$\rightarrow$ ? Er geht nach Hause und ist dabei schnell.

$\rightarrow$ Sein/Das Gehen nach Hause ist schnell.

Wir wollen zunächst davon ausgehen, dass die vom Verb geforderten Ereignisbeteiligten als Bezugsreferenten für die referentenorientierten ereignisinternen Adjunkte dienen können. Hierbei ist sowohl offener als auch begrenzter Bezug möglich. Beim offenen Bezug legt die Form des ereignisinternen Adjunkts den Bezug nicht fest, wie etwa bei den Adjektiven und Partizipien im Deutschen:

Er grüßte die Nachbarin ungekämmt.

$\rightarrow \ldots$ und die Nachbarin war dabei ungekämmt.

$\rightarrow \ldots$ und er war dabei ungekämmt.

Beim begrenzten Bezug legt die Form eines Depiktivs dagegen den Bezug eindeutig fest, so etwa bei den Genitivphrasen im Deutschen, die als ereignisinterne Adjunkte immer Subjektbezug aufweisen:

(6) Er küsste sie voller Frende.

$\rightarrow$... und er war dabei voller Freude.

$\rightarrow \ldots$ ? und sie war dabei voller Freude.

\subsubsection{Aktivitätsorientierte ereignisinterne Adjunkte}

Bei den aktivitätsorientierten ereignisinternen Adjunkten ${ }^{11}$ handelt es sich um Urteile „des Sprechers über den Handlungsvollzug durch das agentische Subjekt" (Pittner 1999, S. 95). Mit anderen Worten: Das aktivitätsorientierte Adverbial beschreibt die Art und Weise, wie das Agens die Handlung ausführt. Bartsch (1976, S. 156) demonstriert dies anhand des folgenden Beispiels und seiner Bedeutungen:

(7) Peter schreibt sorgfältig.

(7a) $\rightarrow$ Peters Schreiben ist sorgfältig.

(7b) $\rightarrow$ Peters Schreib-Handeln ist sorgfältig.

(7c) $\rightarrow *$ Der Schreib-Vorgang ist sorgfältig.

Sorgfältig ist ein Adverbial der Art und Weise, entsprechend ist (7a), wo der Vorgang Schreiben nicht auf seine einzelnen Dimensionen hin differenziert ist, zutreffend. Ebenso ist (7b) zutreffend, wo die Ausführungsdimension differenziert ist. (7c) dagegen, wo die Ausführungsdimension ausgeschlossen ist und allein der Verbalvorgang modifiziert werden soll, ist als Paraphrase von (7) nicht akzeptabel.

11 „Agentive-orientated“ bei Platt/Platt (1972), „predicated of the performance of the action“ $\left(\mathrm{MOD}_{2}\right)$ bei Bartsch (1976, S. 56), ,controller-oriented“ bei Dik et al. (1990, S. 30). 
1.2.3 Vorgangsorientierte ereignisinterne Adjunkte

Umgekehrt modifizieren die vorgangsorientierten ereignisinternen Adjunkte ausschließlich den Verbalvorgang selbst und lassen die Lesart der Aktivitätsorientierung nicht zu. ${ }^{12}$ So ist im folgenden Beispiel (8) neben (8a) die unmittelbar vorgangsorientierte Lesart (8b) zutreffend, die aktivitätsorientierte (8c) jedoch nicht:

(8) Peter redet leise.

(8a) $\rightarrow$ Peters Reden ist leise.

(8b) $\rightarrow$ Der Rede-Vorgang ist leise.

(8c) $\rightarrow$ *Peters Rede-Handeln ist leise.

\subsubsection{Wirkungsorientierte ereignisinterne Adjunkte}

Neben den genannten Subgruppen werden in der Literatur weitere Vorschläge für Gruppeneinteilungen gemacht, die ich als „wirkungsorientiert" zusammenfasse. Das wirkungsorientierte ereignisinterne Adjunkt beschreibt die effizierende oder affizierende Wirkung des Verbalvorgangs auf explizite oder auch implizite Ereignisbeteiligte. ${ }^{13}$ Als Paraphrase können wir nach Bartsch (1976, S. 152) von einer Konstruktion ausgehen, in der ein konsekutiver Satz die Wirkung beschreibt und eine verweisende Pro-Form den Platz des Adverbials im Ausgangssatz einnimmt:

(10) Sie stellte die Bücher ordentlich in den Schrank.

$\rightarrow$ Sie stellte die Bücher so in den Schrank, dass sie geordnet waren.

(11) Max schreibt den Brief völlig unleserlich.

$\rightarrow$ Max schreibt den Brief so, dass er völlig unleserlich ist.

(12) Max hat mir völlig unleserlich geschrieben.

$\rightarrow$ Max hat mir so geschrieben, dass ich es nicht lesen konnte.

\subsubsection{Umstandsorientierte ereignisinterne Adjunkte}

Einen Vorschlag für eine weitere Gruppe machen Zifonun/Hoffmann/Strecker (1997, S. 1205). Für den Orientierungstyp, den kirchlich, schriftlich und auch katholisch in (13) vertreten, wird die Bezeichnung ,,mediativ“" gebraucht: ${ }^{14}$

12 „Verbal-orientated“ bei Platt/Platt (1972), „state-of-the-art oriented“ bei Dik et al. (1990, S. 30), ,MOD ${ }_{0}$ “ bei Bartsch (1976, S. 152).

13 Nach Bartsch geben diese Adjunkte eine, indirect characterization of the consequences arising from the manner in which the process or action is performed" (1976, S. 152) wieder. Bei Dik et al. (1990) wird diese Orientierung als ,goal-orientation“ beschrieben; Platt/Platt (1972) unterscheiden dagegen zwischen drei Gruppen, nämlich „,experiencer orientated“, „result orientated“ und ,factitive orientated“.

14 Es ist möglicherweise symptomatisch für die Komplexität der Differenzierungen, dass katholisch dabei von den Autoren übersehen wird. Es handelt sich jedoch um den gleichen Typ. 
(13) Ich wurde richtig böse, als ich erfuhr, daß man dorthin mußte, bevor man kirchlich getraut werden konnte, und als Marie dann noch davon anfing, daß ich mich schriftlich verpflichten müsse, unsere Kinder katholisch zu erziehen, bekamen wir Streit. [Zifonun/Hoffmann/Strecker (1997, S. 1206)]

Auch ein Adverbial wie chinesisch in (14) gehört in diese Gruppe und ebenso Adjunkte wie in (15), die angeben, in welcher Sprache ein Vorgang stattfindet:

(14) Wir haben gestern chinesisch gegessen.

(15) Mitarbeiter des Landratsamts Breisgau-Hochschwarzwald und der Kaysersberger Verwaltungsgemeinschaft erörtern auf Französisch und Deutsch ihre kommunale Umweltschutz-, Tourismus- oder Kulturpolitik.

Diese Adjunkte weisen eine gewisse Nähe zu den aktivitätsorientierten ereignisinternen Adjunkten auf, indem sie ebenfalls die Ausführungsdimension beschreiben. Es wird jedoch nicht der unmittelbare Handlungsvollzug modifiziert, sondern die Handlung wird in Bezug auf Umstände modifiziert, die den Vollzug klassifizieren. ${ }^{15}$ Ich nenne die Gruppe „umstandsorientiert“:

(13a) $\rightarrow$ Man wird getraut, wobei die Umstände der Trauung kirchlich sind. $\rightarrow$ Ich muss mich verpflichten, wobei die Umstände der Verpflichtung schriftlich sind.

$\rightarrow$ Ich erziehe unsere Kinder, wobei die Umstände der Erziehung katholisch sind.

(14a) $\rightarrow$ Wir haben gegessen, wobei die Umstände des Essens chinesisch waren.

(15a) $\rightarrow$ Mitarbeiter ... erörtern ..., wobei die Umstände der Erörterung auf Französisch und Deutsch sind.

\subsubsection{Doppelte Orientierung und Vagheit der Orientierung}

Die Prädikation eines referentenorientierten ereignisinternen Adjunkts kann mehr oder weniger eng mit dem Verbalvorgang zusammen hängen. In einem Satz wie das Paket kam unversehrt an verändert die Unversehrtheit des Pakets nichts an dem Vorgang des Ankommens selbst. Unversehrt ist in diesem Satz also ein ausschließlich referentenorientiertes ereignisinternes Adjunkt. Voller Frende in Er kë̈ste sie voller Freude (siehe (6)) charakterisiert dagegen sowohl den Referenten des Subjekts als auch die Handlung. ${ }^{16}$ Es ist doppelt

\footnotetext{
15 Einem anonymen Gutachter verdanke ich den Hinweis, dass diese Art der Modifikation dem Typ der „klassifikatorischen Modifikation“ (vgl. Rijkhoff 2004) im Bereich der Nominalattribution entspricht.

16 Und genau dies ist wahrscheinlich auch der Grund der ausschließlichen Subjektorientierung bei den Genitiven, siehe (6).
} 
orientiert. Ebenso können aktivitäts-, wirkungs- und vorgangsorientierte gleichzeitig referentenorientiert sein. So drückt eilig in er trank eilig seinen Tee aus eine durch das Agens verursachte und von ihm kontrollierte Aktivität aus; es ist also aktivitätsorientiert. Aktivitätsorientierte ereignisinterne Adjunkte sagen immer auch etwas über den Zustand des Agens bei der Handlungsausführung aus. Das bedeutet jedoch keine Ambiguität oder Vagheit zwischen einer vorgangs- und einer referentenorientierten Lesart bei eilig, sondern die referentenorientierte Semantik ergänzt die Vorgangsorientierung. ${ }^{17}$

Doppelte Orientierung liegt auch bei einer Gruppe von Adverbialen im Türkischen vor, den strukturell in die Sprache integrierten, adverbial gebrauchten Ideophonen. Sie sind vorgangsorientiert, indem sie das Verb deskriptiv modifizieren und häufig auch Kollokationen mit bestimmten Verben bilden (z.B. şırl şrml, etwa: „plätschernd“, das immer mit ak-- „fließen“ zusammen auftaucht). Ihre Selektion ist jedoch wesentlich von den semantischen Eigenschaften des Agens (in intransitiven Sätzen) bzw. des Patiens (in transitiven Sätzen) in der jeweiligen Äußerung motiviert, oder sie sagen etwas über die physische Verfassung eines nominalen Referenten aus. So verweist z.B. çatır catır auf das Verbrennen, Zerbrechen, Herausgerissen- oder Zusammengedrückt-Werden eines harten Gegenstandes (Jendraschek 2001), und bön bön bak- „dümmlich gucken“ ist über die Modifikation des Verbalvorgangs hinaus ein deskriptiver Kommentar über die psychische Verfassung, in der das Subjekt die Handlung ausführt. Die Tatsache, dass das Türkische derartige Formen aufweist und das Deutsche nicht, hängt eng mit der unterschiedlichen lexikalischen Semantik des Verbs in den beiden Sprachen zusammen. Ich werde in Abschnitt 4.2. darauf zurückkommen.

Während bei den doppelt orientierten Formen zwei Orientierungen gekoppelt auftreten, muss im Prinzip auch davon ausgegangen werden, dass es Formen gibt, bei denen mehrere Verwendungen bzw. Lesarten möglich sind. Auf ein Beispiel einer derartigen uneindeutigen Orientierung weist Bartsch (1976, S. 144) mit (16) hin:

Die Laterne leuchtet hell.

Hell kann einerseits den Test für referentenorientierte ereignisinterne Adjunkte bestehen ( $\rightarrow$ Die Laterne leuchtet. Sie ist dabei hell.) Andererseits besteht bell auch den Test für vorgangsorientierte ereignisinterne Adjunkte $(\rightarrow$ der Leucht-Vorgang ist hell). Ich nenne derartige Formen „vage“. Mit „Vagheit“ sollen Mehr- und Uneindeutigkeiten der Orientierung bezeichnet werden. Das kann eine uneindeutige Orientierung eines ereignisinternen Adjunkts in einem spezifischen Satz sein, oder aber die prinzipielle Mehrdeutigkeit einer Form, die sich in einem spezifischen Satzkontext in die eine oder andere Orientierung auflösen kann.

17 Ähnlich auch z.B. träge gegenüber langsam, vgl. Zifonun/Hoffmann/Strecker (1997, S. 1199). 


\section{Befragung der Daten}

Die bisher entwickelten Begrifflichkeiten beanspruchen übereinzelsprachliche Gültigkeit. Das heißt aber eben gerade nicht, dass eine spezifische Funktions-Form-Beziehung übereinzelsprachlich ist. Die kontrastive Forschungsfrage lautet also:

- Welche Formen haben ereignisinterne Adjunkte (hier: Depiktive und Adverbiale der Art und Weise) im Türkischen und Deutschen?

- Wieweit korrelieren Form und semantische Orientierung bei den ereignisinternen Adjunkten (hier: Depiktive und Adverbiale der Art und Weise)?

Diese Fragen werden im Folgenden in Bezug auf die beiden zu kontrastierenden Sprachen abgearbeitet. Ich gehe dabei von den Formen aus; sie werden vorgestellt und auf ihre semantische Orientierung hin überprüft. Die türkischen Beispiele werden noch um einige Erläuterungen ergänzt.

\subsection{Deutsch}

\subsubsection{Adjektive und Partizipien}

Die häufigste Form von ereignisinternen Adjunkten im Deutschen sind Adjektive und Partizipien (Partizip I und II) des folgenden Typs:

(17) ... setzte sich müde/schräg/verwnndert/ ungekë̈sst/bektisch hinter das Steuer.

(18) ... fotografiert ihn immer nackt/ verwackelt/ liebevoll.

(19) Sie sind schonend getrocknet. [Packungsaufschrift]

(20) Bitte geben Sie die Flasche gespïlt/ausgeschlafen/tanæend zurück.

Die Beispiele machen deutlich, dass diese Formen nicht prinzipiell auf eine Orientierung festgelegt sind. Vielmehr geschieht die Festlegung lexikalisch. Eine Ausnahme bilden allerdings denominale Adjektive auf -lich und -isch, die immer umstandsorientiert sind:

(21) ... wurde psychologisch beraten

(22) ... hat chinesisch gekocht

\subsection{2 als-Phrasen}

Immer referentenorientiert sind Phrasen mit dem Junktor als. Sie können eine temporäre Funktion oder Rolle, oder auch eine Lebensphase des Bezugsreferenten prädizieren: 
(23) ... Elsa, die als Kellnerin in einer Weinstube bediente,

(24) Als kleiner Junge habe ich einmal durch ein Schlüsselloch beobachtet, wie ...

\subsubsection{Vergleiche mit wie}

Vergleiche mit wie sind immer aktivitäts- oder vorgangsorientiert:

... mischt die Karten wie ein erfahrener Spieler

(26) ... raste um die Ecke wie ein geölter Blitz.

\subsubsection{Präpositionalphrasen}

Eine ebenfalls häufige Form von ereignisinternen Adjunkten im Deutschen sind Präpositionalphrasen. Stativ-lokativische Präpositionalphrasen mit in, auf, unter, vor oder hinter sind in der Regel referentenorientiert:

(27) der Kunde liefert seine Kleider in einem persönlichen Kleidersack ab und erhält sie später im gleichen Sack sauber zurück

Kombinationen einiger dieser Präpositionen mit spezifischen Komplementen können spezifische andere Orientierungen bewirken. So sind Präpositionalphrasen mit in oder auf und einer Sprachenbezeichnung umstandsorientiert, und Präpositionalphrasen mit unter und einem Komplement, das einen Vorgang bezeichnet, sind vorgangs- oder referentenorientiert:

(28) ... grüßte mich auf Französisch

(29) ... verließ unter Protest den Gerichtssaal

(30) Der Wagen raste unter Applaus auf die Zielgerade zu.

Präpositionalphrasen mit mit oder obne sind aktivitäts- oder referentenorientiert:

(31) bitte gespült und mit Verschluss/mit Vorsicht/mit Schwung zurückgeben

(32) flog mit Koffer/ohne Handgepäck einmal um die Welt

(33) ... war schon mit fünfžehn Jahren eine Berühmtheit

(34) Die Feuerwehr war mit fünf Mann im Einsatz.

\subsection{5 „Explizitphrasen“}

Ein gesondert zu behandelnder Typ von ereignisinternen Adjunkten in der Form von Präpositionalphrasen sind Formen, bei denen das Komplement als Kernnomen ein Substantiv wie Zustand, Haltung, Art und Weise, Form oder 
Stil enthält, während ein voranstehendes Attribut den semantischen Kern des Adjunkts enthält. Sie sind dem formellen Register des Deutschen zuzuordnen. Ich nenne sie hier „Explizitphrasen“, da das Kernnomen vor allem die Aufgabe zu haben scheint, die Orientierung des ereignisinternen Adjunkts zu explizieren. Explizitphrasen mit der Präposititon in und Zustand und in und Haltung scheinen immer referentenorientiert, vgl. (35) und (36), Explizitphrasen mit auf ... (Art und) Weise sind vorgangs-, aktivitäts- oder wirkungsorientiert, vgl. (37), Explizitphrasen mit in ... Form sind wirkungsorientiert, vgl. (38) und Explizitphrasen mit in ... Stil sind aktivitätsorientiert, vgl. (39):

... warf in alkobolisiertem Zustand eine Scheibe ein

(36) Aber sie sagte in unveränderter Haltung: „Verzeihen Sie, ...“

(37) ... hat mich auf seltsame/liebevolle/erschreckende/elektrisierende/ elektrisierte Art und Weise angeschaut

(38) Der Vertrag wurde in einer für beide Seiten annehmbaren Form aufgelöst.

(39) Die Muster wurden in einem spezifischen Stil hineingewoben.

2.1.6 Spezialisierte Formen, geschlossene Gruppen, idiomatisierte Formen

Eine als idiomatisiert zu bezeichnende weitere Form von ereignisinternen Adjunkten sind Nominalphrasen im Genitiv mit dem Attribut voller und einem Kernnomen, das ein Gefühl bezeichnet. Sie sind aktivitäts- oder referentenorientiert:

... warf sich ihm voller Freude/voller Inbrunst/voller Scham an die Brust

Ebenfalls idiomatisiert sind Reduplikationen von Körperteilbezeichnungen, die als Zwischenglied die Präposition in oder an haben. Sie geben als referentenorientierte ereignisinterne Adjunkte Körperkontakte zwischen den pluralischen Bezugsreferenten an:

(41) Hand in Hand, Schulter an Schulter, Kopf an Kopf

Dann gibt es noch eine kleine Gruppe von Adverbien, bei denen die Referentenorientierung lexikalisch festgelegt scheint: ${ }^{18}$

\section{barfu $\beta$, (mutterseelen)allein}

Einen formal spezifizierten Bereich bilden zudem quantifizierende referentenorientierte Adjunkte mit $₹ u$ und Mann hoch, die sich immer auf belebte Referenten beziehen:

$18 \quad$ Vgl. Renz/Hentschel (2011). 
(43) sie stürmten zu dritt aus dem Haus

(44) sie stürmten drei Mann hoch aus dem Haus

\subsection{Türkisch}

\subsection{1 olarak}

Das Türkische bildet zum einen ereignisinterne Adjunkte mit dem postponierten Junktor olarak. olarak ist die (erstarrte) Konverbform des Kopulaverbs of- „sein“ mit dem -ArAk-Konverb. Es wird auch zur Bildung von anderen semantischen Typen von Adjunkten verwendet. Als ereignisinternes Adjunkt bildet es vorwiegend referentenorientierte Formen:

kurmay yüzbaş olarak görev-e başla-dı
Generalstab Hauptmann JUNK Dienst-DAT beginnen-PRÄT(3SG)
„Er begann seinen Dienst als Hauptmann des Generalstabs.“

(46) Öğretmen-ler okul-a hazırlıkl olarak gel-meli-dir-ler. Lehrer-PL Schule-DAT vorbereitet JUNK kommen-OBLIG-FAKT-PL „Die Lehrer müssen vorbereitet zur Schule kommen.“

Mit Sprachenbezeichnungen als Komplement kann olarak auch umstandsorientierte ereignisinterne Adjunkte bilden:

$$
\begin{aligned}
& \text {... bizi Fransiz̧a olarak selamla-di. } \\
& \text { uns(AKK) Französisch JUNK grüßen-PRÄT(3SG) } \\
& \text { „,... sie begrüßte uns auf Französisch.“ }
\end{aligned}
$$

\subsubsection{Denominale adverbiale Ableitung mit -CA}

Ausschließlich aktivitätsorientierte ereignisinterne Adjunkte bilden denominale adverbiale Ableitungen mit dem Suffix $-C A$ :

Saliha onu bir süre sessiz̨-ce dinle-di.

S. ihn(AKK) eine Zeitlang ohne.Laut-ADV zuhören-PRÄT(3SG)

„Saliha hörte ihm eine Zeitlang schweigend zu."

$\begin{array}{llll}\text { biri } & \text { erkek-çe } & \text { el-in-i } & \text { öp-üp... } \\ \text { eine/r } & \text { Mann-ADV } & \text { Hand-POSS.3SG-AKK } & \text { küssen-KONV } \\ \text { „wenn einer männlich ihre Hand küsst, ... “ }\end{array}$

Hinzuzufügen ist, dass - $C A$ mit bestimmten Phrasenrestriktionen auch die Agensphrase in Passivsätzen bilden kann. 
2.2.3 Reduplikationen als adverbiale Ableitungsstrategie

Eine weitere denominale Strategie zur Bildung von Adverbialen sind Reduplikationen. Sie bilden zum einen referentenorientierte ereignisinterne Adjunkte:

$\begin{array}{lllll}\text { Bunlar1 } & \text { kus-ana } & \text { dek } & c i g \breve{g} c i \breve{g} & \text { ye-r-im. } \\ \text { dies(AKK) } & \text { erbrechen-KONV } & \text { bis } & \text { roh(REDUPL) } & \text { essen-AOR-1SG } \\ \text { „Die esse ich roh bis zum Erbrechen.“ } & \end{array}$

Reduplikationen können jedoch auch eine Vorgangsorientierung haben. Diese verbindet sich immer mit einer Modifizierung der Aktionalität im Sinne einer Dauer oder Iterativität:

$\begin{array}{llll}\begin{array}{l}\text { herkes rahat rahat } \\ \text { jeder }\end{array} \text { ruhig(REDUPL) } & \text { git-ti } & \text { oy-un-u } \\ \text { kullan-d1 } & & \\ \text { benutzen-PRÄT(3SG) } & \text { Stimme-POSS.3SG-AKK } \\ \text {,in aller Rube ging jedermann hin und wählte“ }\end{array}$

Öyle sessiz, sessiz. otur-uyor mu-ydu-n orada? So leise(REDUPL) sitzen-PROGR QUE-PRÄT-2SG dort (LOK) „Hast du da so still (herum-)gesessen?“

Die Verwendung derartiger Formen gehört eher dem informellen Register an.

\subsubsection{Ideophone}

Ebenfalls zum informellen Register gehören Ideophone, von denen es mehrere Bildungsformen gibt. Zum einen ist da die einfache Reduplikation des Stamms, z.B. lop lop, zum zweiten die Reduplikation der um das Suffix -Ir erweiterten Stammform, z.B. lopur lopur, zum dritten die mit der Zitierform diye verbundene Stammform. Außer der einfachen Stammreduplikation sind die Formen jedoch nur begrenzt produktiv, und einige Ideophone haben zusätzlich auch noch Sonderformen, wie in dem Fall von lop, das auch noch zu loppadak werden kann. Sie sind immer vorgangsorientierte ereignisinterne Adjunkte, und die Vorgangsorientiertheit verbindet sich mit einer Modifizierung der Aktionalität im Sinne einer Dauer oder Iterativität. Die unterschiedlichen Bildungsformen führen zudem zu einer unterschiedlichen deskriptiven Modifizierung des Verbalvorgangs. So lassen sich im untenstehenden Beispielsatz alle vier genannten Variationen von lop einsetzen:

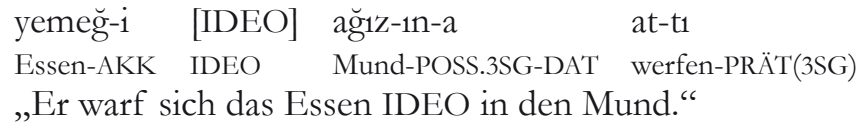


lop lop und lopur lopur würden hier den Verbalvorgang als „mehrfach/andauernd" modifizieren, wobei lopur lopur im Gegensatz zu lop lop eine längere Dauer der einzelnen Handlungselemente impliziert. lop diye würde dagegen die einmalige Vollendung der Handlung implizieren und loppadak eine zusätzliche Plötzlichkeit oder Spontanität.

\subsubsection{Nichtabgeleitete Adjektive}

Der Einsatz nichtabgeleiteter Adjektive ist eine weitere Strategie zur Modifizierung der ereignisinternen Semantik, die das Türkische anwendet. Wie beim Einsatz von Adjektiven als ereignisinterne Adjunkte im Deutschen (vgl. Teil 2.1.1 oben), so sind auch diese Formen nicht prinzipiell auf eine Orientierung festgelegt. Wir finden Referentenorientiertheit $(54,55)$, Vorgangsorientiertheit (56), Aktivitätsorientiertheit (57) und Wirkungsorientiertheit (58):

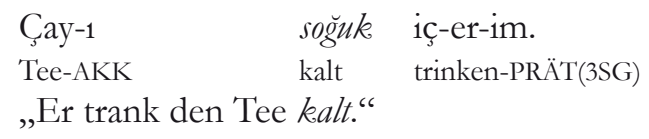

(56) İş-ler kötü gid-iyor.

Sache-PL schlecht gehen-PRÄS „Es läuft schlecht.“

(57) Sözcük-ler-in-i dikekatli seç-er.

Wort-PL-POSS-AKK sorgfältig wählen-AOR(3SG)

„Sie wählt ihre Worte sorgfältig.“

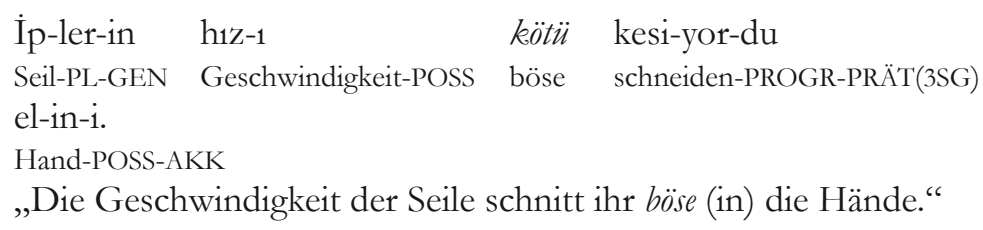

Diese Formen sind allerdings, wie wir in Abschnitt 2.3 unten noch sehen werden, syntaktisch, semantisch und pragmatisch stark eingeschränkt, und dies unterscheidet sie wesentlich von ihren scheinbaren Äquivalenten im Deutschen, die die kanonische Form von ereignisinternen Adjunkten im Deutschen darstellen. 


\subsection{6 „Explizitphrasen“ im Lokativ und Instrumental}

Auf den ersten Blick weist auch eine weitere Form Ähnlichkeiten mit deutschen Konstruktionen auf, nämlich mit den „Explizitphrasen“ (vgl. 2.1.5). Wir haben es bei den türkischen Explizitphrasen mit Nominalphrasen im Lokativ oder Instrumental zu tun, deren Kernnomen mit „Zustand“, „Position“, „Form“, „Haltung“ oder „Stellung“ übersetzt werden kann. Auch hier bildet ein modifizierendes Element den eigentlichen semantischen Kern der Phrase, dies ist entweder ein attributives Adjektiv oder Partizip, oder es ist ein Nomen, das mit dem Kernnomen ein Possessivkompositum bildet.

Das Türkische verfügt über eine breite Palette von „Zustandsnomina“: hal, durum (beide: „Zustand“), şekil, biçim (beide: „Form“), tavr, eda (beide: „Benehmen, Haltung“) und einige mehr. Kasus (Lokativ versus Instrumental) und Wahl des Kernnomens steuern die semantische Orientierung.

Explizitphrasen im Lokativ mit hal, şekil, durum und biçim bilden referentenorientierte ereignisinterne Adjunkte, vgl. (59), (60) und (61), şekil mit Lokativ bildet auch vorgangs- und aktivitätsorientierte Adjunkte, vgl. (62), biçim im Lokativ bildet auch wirkungsorientierte Adjunkte, vgl. (63):

$$
\begin{array}{llll}
\text { Çay-1 kıəğn bir hal-de } & \text { iç-ti. } \\
\text { Tee-AKK wütend ein Zustand-LOK } & \text { trinken-PRÄT(3SG) } \\
\text { „Er trank wütend den Tee.“ } &
\end{array}
$$

$\begin{array}{lll}\text { Eğik durum-da bekl-iyor-du } & \text { denizalt1. } \\ \text { schräg Zustand-LOK } & \text { warten-PROGR-PRÄT(3SG) } & \text { U-Boot } \\ \text { „In schräger Stellung wartete das U-Boot.“ } & \end{array}$

uykeu-sun-u al-ma-mus bir bal-de

Schlaf-POSS.3SG-AKK nehmen-NEG-RESULT ein Zustand-LOK uyan-1r.

aufwachen-AOR(3SG)

„Er wacht unausgeschlafen auf.“

$\begin{array}{lllll}\text { mutfak } & \text { bez-ler-in-i } & \text { canl } & \text { bir } & \text { sekil-de } \\ \text { Küche } & \text { Tuch-PL-POSS-AKK } & \text { energisch } & \text { ein } & \text { Zustand-LOK } \\ \text { silk-ti. } & & & & \\ \text { ausschütteln-PRÄT(3SG) } & & & \end{array}$

„Energisch schüttelte sie ihre frischgewaschenen Küchentücher aus.“

(63) $\mathrm{Bu}$ alışkanlık, küçüklüğ-ün-de bedel-i ağır bir

dies Angewohnheit Kindheit-POSS.SG-LOK Preis-POSS schwer ein biçim-de öde-n-dikten sonra edin-il-miş.

Zustand-LOK bezahlen-PASS-KONV nach bekommen-PASS-EVID

„Diese Angewohnheit soll man bekommen, wenn man in seiner Kindheit auf schmerzhafte Weise dafür den Preis bezahlt hat.“ 
Explizitphrasen im Instrumental werden mit hal, tavr und eda als Kernnomen gebildet. Mit hal und Instrumental gebildete ereignisinterne Adjunkte sind dabei immer referentenorientiert, zudem muss der Bezugsreferent das Subjekt sein und es besteht eine mit dem Possessiv markierte Personenkongruenz zwischen dem Explizitnomen und dem Subjekt:

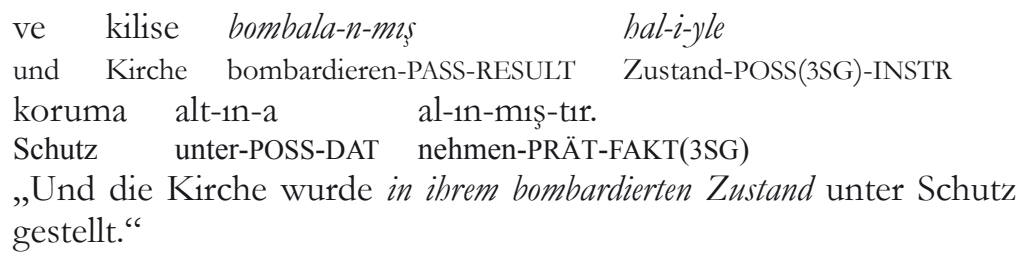

Mit $e d a$ oder auch tavr (beide: „Benehmen, Haltung ${ }^{6}$ ) und Instrumental gebildete ereignisinterne Adjunkte sind immer aktivitätsorientiert:

\begin{tabular}{|c|c|c|c|}
\hline Nişanl1-m & mazlum & bir & eda-yla (...) \\
\hline $\begin{array}{l}\text { Verlobte-POSS.1SG } \\
\text { sor-ar-d1. }\end{array}$ & bescheiden & ein & Benehmen-INSTR \\
\hline
\end{tabular}

\subsubsection{Andere Nominalphrasen mit Lokativ und Instrumental}

Auch außerhalb von Explizitphrasen können der Instrumental- und der Lokativkasus ereignisinterne Adjunkte bilden. Wie bei den Explizitphrasen mit Instrumental (siehe oben), so ist auch bei allen anderen referentenorientierten ereignisinternen Adjunkten, die mit Instrumental gebildet werden, nur das Subjekt als Bezugsreferent möglich, und das Adjunkt muss Possessivkongruenz mit dem kontrollierenden Subjekt aufweisen:

$\begin{array}{llll}\text { denizkız1 } & \text { çplak } & \text { meme-ler-i-yle } & \text { yan-1n-a } \\ \text { Meeresjungfrau } & \text { nackt } & \text { Brust-PL-POSS(3SG)-INSTR } & \text { Seite-POSS-DAT }\end{array}$

sokul-ur.

anschmiegen-AOR(3SG)

„die Meerjungfrau schmiegt sich mit ibren nackten Brüsten an seine Seite.“

Darüber hinaus können Nominalphrasen mit Instrumental aktivitätsorientierte ereignisinterne Adjunkte bilden:

$$
\begin{array}{llll}
\text { Hirs-la, } & \text { bınc-la } & \text { sarıl-d1-m } & \text { makine-ye. } \\
\text { Ärger-INSTR } & \text { Hass-INSTR } & \text { angreifen-PRÄT-1SG } & \text { Maschine-DAT } \\
\text { „Wütend und hasserfüllt attackierte ich die Maschine.“ }
\end{array}
$$


Nominalphrasen im Lokativ sind als ereignisinterne Adjunkte immer referentenorientiert. Dabei spezialisieren sie sich auf semantische Bereiche wie die Modifikation von Ort und Alter:

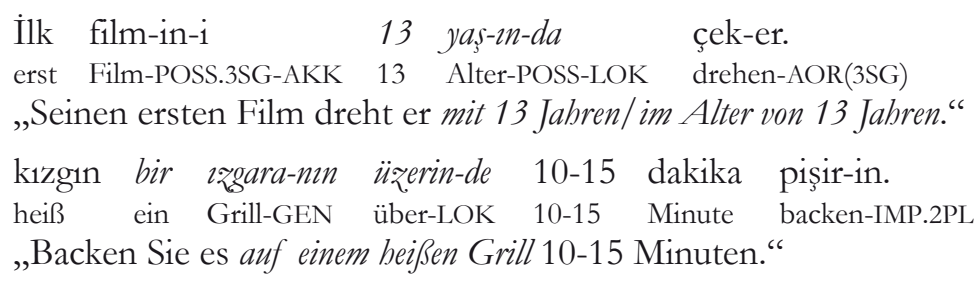

\subsubsection{Vergleiche}

Vergleiche mit der Postposition gibi sind immer aktivitäts- oder vorgangsorientiert:

$$
\begin{array}{lllll}
\text { (70) Simssek gibi sırı̆-1 } & \text { el-in-den } & \text { kap-tı. } \\
\text { Blitz } & \text { wie } & \text { Stange-AKK } & \text { Hand-POSS.3SG-ABL } & \text { reißen-PRÄT(3SG) } \\
\text { "Wie ein Blitz riss er ihr die } & \text { Stange aus der Hand.“ } \\
\text { (71) Tas gibi dur-ur-lar } & \text { öyle. } & \\
\text { Stein wie sitzen-AOR-PL } & \text { so } & \\
\text { "Wie Steine sitzen sie da.“ } &
\end{array}
$$

\subsubsection{Reduzierte Konverbsätze}

In Abschnitt 1.1 wurde festgelegt, dass wir uns hier auf nicht satzwertige ereignisinterne Adjunkte konzentrieren. Türkisch ist eine Sprache, die (hier: adverbiale) Subordination ausschließlich mit nichtfiniten Konverbphrasen realisiert, und so müssen wir auch im Umfeld reduzierter Konverbphrasen ${ }^{19}$ nach Kandidaten für ereignisinterne Adjunkte suchen. Es finden sich drei Formen. Zunächst sei das Konverb -ken aufgeführt. Reduzierte Adjunktsätze mit -ken bilden referentenorientierte ereignisinterne Adjunkte, und sie sind dabei den Lokativphrasen (siehe 2.2.7) ähnlich, indem sie sich auf semantische Bereiche wie die Prädikation von Lebensspanne, Alter und Zeit spezialisieren. Es ist Koreferenz mit dem Subjekt, dem direkten (Akkusativ-) und dem indirekten (Dativ-)Objekt des übergeordneten Satzes möglich.
(72) kağıt oyun-lar-1 oyna-ma-y1 sev-er-di-m
Karte Spiel-PL-POSS spielen-NOM-AKK lieben-AOR-PRÄT-1SG genc-ken.
jung-KONV
„Als Kind/als ich jung war liebte ich es, Kartenspiele zu spielen.“

19 Als „Reduzierte Konverbphrasen“ bezeichne ich entsprechend der in Abschnitt 1.1 genannten Festlegungen solche Konverbphrasen, die keine eigenständigen satzprosodischen Einheiten bilden. 
(73) Eş-i ve kayınbirader-in-i uyku-da-yken öldür-dü.

Frau-POSS und Schwager-POSS-AKK Schlaf-LOK-KONV töten-PRÄT(3SG) „Er tötete seine Frau und seinen Schwager im Schlaf/während sie schliefen."

Reduzierte Sätze, die mit dem Konverb - ArAk gebildete sind, bilden referenten-, aktivitäts- und vorgangsorientierte ereignisinterne Adjunkte. Koreferenz ist hier nur mit dem Subjekt des übergeordneten Satzes möglich:

(74) davranış-lar-1n-1 üzül-erek izle-mek-te-yim.

Benehmen-PL-POSS-AKK traurig-KONV beobachten-INF-LOK-1SG

„Ich beobachte traurig ihr Verhalten.“

$\begin{array}{ll}\text { yürü-yerek } & \text { uzaklaş-t1-lar } \\ \text { laufen-KONV } & \text { sich.entfernen-PRÄT-PL }\end{array}$

„Sie entfernten sich laufend/sie gingen weg."

Das reduplizierte Konverb $-A-A$ bildet aktivitäts- oder vorgangsorientierte ereignisinterne Adjunkte. Wie bei den anderen reduplizierten ereignisinternen Adjunkten (siehe 2.2.3), so geht auch hier damit eine aktionale Modifizierung der Dauer oder Wiederholung einher. Koreferenz des Subjekts des Adjunktsatzes ist nur mit dem Subjekt des übergeordneten Satzes möglich:

$\begin{array}{llll}\text { Yürü-ye yürü-ye } & \text { Taşhan'-1n } & \text { kap1-Sin-a } & \text { gel-miş-im. } \\ \text { laufen-REDUPL.KONV } & \text { T.-GEN } & \text { Tür-POSS-DAT } & \text { kommen-EVID-1SG } \\ \text { „In einem fort laufend bin ich bis zur Tür von Taşhan gekommen." }\end{array}$

\subsubsection{Nichtproduktive Formen}

Abschließend sind zwei kleinere geschlossene Gruppen von ereignisinternen Adjunkten zu nennen. Dies sind zum einen nominale Reduplikationen mit Dativ, die als idiomatisierte Formen immer referentenorientiert sind und hier wie bei ihrem deutschen Äquivalent (vgl. Abschnitt 2.1.6) einen meist körperlichen Kontakt zwischen belebten Pluralreferenten angeben, z.B. el el-e (Hand Hand-DAT) „Hand in Hand“, omuz omuz-a (Schulter Schulter-DAT) „Schulter an Schulter“, aber auch: nefes nefes-e (Atem Atem-DAT) „außer Atem“. Und dann gibt es wie im Deutschen auch im Türkischen noch eine kleine Gruppe von Adverbien, bei denen die Referentenorientierung lexikalisch festgelegt scheint, u.a. yahnayak „,barfuß“, tek bas-POSS-DAT „allein“, beraber „zusammen“, ayakta ,stehend“, und einige weitere Adverbien der Körperhaltung.

\subsection{Einordnende Erläuterungen}

Bevor wir zu einer zusammenfassenden Beschreibung und Bewertung der deutsch-türkischen Kontraste bei den ereignisinternen Adjunkten kom- 
men, müssen noch zwei einordnende Erläuterungen zum morphosyntaktischen Verhalten einiger der in 2.1 und 2.2 aufgeführten Formen gemacht werden. Diese betreffen Kontraste bei zwei auf den ersten Blick ähnlichen Formen im Türkischen und Deutschen, nämlich den „Explizitphrasen“ (siehe die Abschnitte 2.1.5 und 2.2.6) und den Adjektivphrasen (2.1.1 und 2.2.5).

In Abschnitt 2.1.5 wurde bereits darauf hingewiesen, dass die Explizitphrasen im Deutschen nicht grammatisch, sondern stilistisch motiviert sind, indem sie - vornehmlich im formalen schriftsprachlichen Register - die Orientierung des ereignisinternen Adjunkts explizieren. Entsprechend ist als Alternative zu der Präpositionalphrase mit dem „Explizitnomen“ als Kern des Komplements die Setzung der Adjektiv- oder Partizipialphrase immer grammatisch wohlgeformt:

(35a) warf in alkoholisiertem Zustand eine Scheibe ein $\rightarrow$ warf alkoholisiert eine Scheibe ein

(36a) aber sie sagte in unveränderter Haltung: ,Verzeihen Sie, ...“ $\rightarrow$ aber sie sagte unverändert. „Verzeihen Sie, ..."

(38a) der Vertrag wurde in einer für beide Seiten annehmbaren Form aufgelöst $\rightarrow$ der Vertrag wurde für beide Seiten annehmbar aufgelöst

Diese stilistische Motivierung unterscheidet die Explizitphrasen im Deutschen von den in Abschnitt 2.2.6 beschriebenen türkischen Explizitphrasen. Bei letzteren nämlich ist die Alternative einer Setzung der Adjektivoder Partizipialphrase grundsätzlich ungrammatisch:

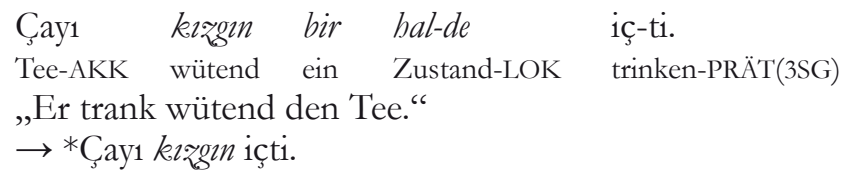

(60a) Eğik durum-da bekl-iyor-du denizaltı. schräg Zustand-LOK warten-PROGR-PRÄT U-Boot „In schräger Stellung wartete das U-Boot.“ $\rightarrow{ }^{*}$ Eğik bekliyordu denizaltı.

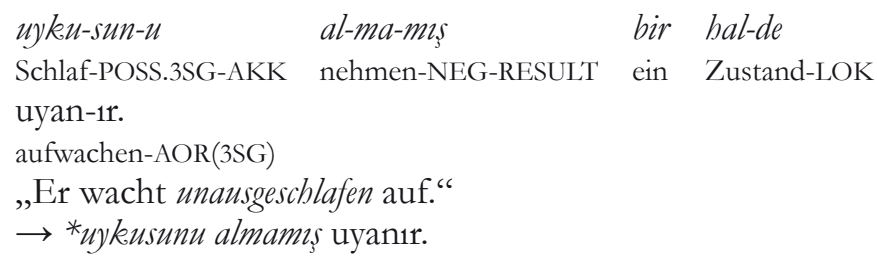

Die türkischen Explizitphrasen sind also nicht stilistisch, sondern grammatisch motiviert: Der adverbiale Einsatz einer kasusmarkierten Nominalphrase ist grammatisch wohlgeformt, der eines Adjektivs oder eines Partizips ist es dagegen nicht. 
Wenn dies so ist, dann drängt sich die Frage auf, wie die in Abschnitt 2.2.5 aufgeführten Beispiele von nichtabgeleiteten Adjektiven im Türkischen zu analysieren sind. Tatsächlich müssen wir auch den adverbial verwendeten Adjektiven und Partizipien im Deutschen und im Türkischen jeweils unterschiedlichen morphosyntaktischen Status zusprechen: Im Deutschen erscheinen diese als vollwertige Adverbiale; es handelt sich um die bevorzugte, grammatisch unbeschränkte Form von ereignisinternen Adjunkten in der Verbalphrase. Im Türkischen dagegen sind die Formen höchst eingeschränkt. Einerseits handelt es sich hier um Wortstellungsbeschränkungen: Die nichtabgeleiteten Adjektive dürfen die unmittelbar präverbale Position nicht verlassen, während alle anderen Formen von ereignisinternen Adjunkten keinen solchen syntaktischen Beschränkungen unterliegen:

\begin{tabular}{|c|c|c|c|}
\hline Aa) ? & $\begin{array}{l}\text { Çay-1 } \\
\text { Tee-AKK }\end{array}$ & $\begin{array}{l}\text { iç-ti } \\
\text { trinken-PRÄD(3SG) }\end{array}$ & $\begin{array}{l}\text { soğnk. } \\
\text { kalt }\end{array}$ \\
\hline 10 & $\begin{array}{l}\text { Dikekath } \\
\text { sorgfältig }\end{array}$ & $\begin{array}{l}\text { sözcük-ler-in-i } \\
\text { Wort-PL-POSS.3SG-AKK }\end{array}$ & $\begin{array}{l}\text { seç-er. } \\
\text { wählen-AOR (3SC }\end{array}$ \\
\hline
\end{tabular}

Zum zweiten unterliegen die referentenorientierten nichtabgeleiteten Adjektive auf interessante Art und Weise den Valenzrestriktionen des Verbs, in dessen Phrase sie stehen, indem sie mit transitiven Verben ausschließlich Objektbezug haben können:

$\begin{array}{llll}\text { (54b) } & \text { Çay-1 } & \text { soğık } & \text { iç-ti. } \\ & \text { Tee-AKK } & \text { kalt } & \text { trinken-PRÄT(3SG) } \\ & \text { „Er trank den Tee kalt“ } \\ \text { (54c) } & \text { *Çay-1 yorgun } & \text { iç-ti } \\ & \text { Tee-AKK müde } & \text { trinken-PRÄT(3SG) } \\ & \text { „Er trank den Tee müde.“ }\end{array}$

Es scheint zudem, dass die Gruppe der Adjektive, die in dieser Form verwendet werden können, sich auf ein Inventar von Adjektiven beschränkt, die basale Geschwindigkeiten (hıæh „schnell", yavas "langsam") und basale Bewertungen (iyi ,gut", kötü ,schlecht") einer Handlungsausführung modifizieren, oder auch referentenorientierte Modifikationen liefern, die erwartbare Zustände der Bezugsreferenten im Rahmen der Handlung prädizieren (man trinkt Tee „heiß“ oder „kalt", man kann etwas „wütend“ oder ,traurig“ fragen, siehe (55)). Modifikationen, die außerhalb dieser basalen Kategorien liegen, sind mit den nichtabgeleiteten Adjektiven nicht leistbar:

(54d) Çay1 soğuk/hræ̨l/ iyil kötü içti. „Er trank den Tee kalt/schnell/gut/schlecht.“

(54e) Çay1 ?demli/?sesli/?sessiz/içti „Er trank den Tee stark gerogen/laut/leise.“ 
Auch Erweiterungen der nichtabgeleiteten Adjektive müssen sich im Rahmen basaler Kategorien (cok, „sehr", biraz, „ein wenig“) bewegen - wobei nicht ganz klar ist, ob es sich hierbei um eine semantische Restriktion oder eine Phrasenrestriktion oder um beides handelt:

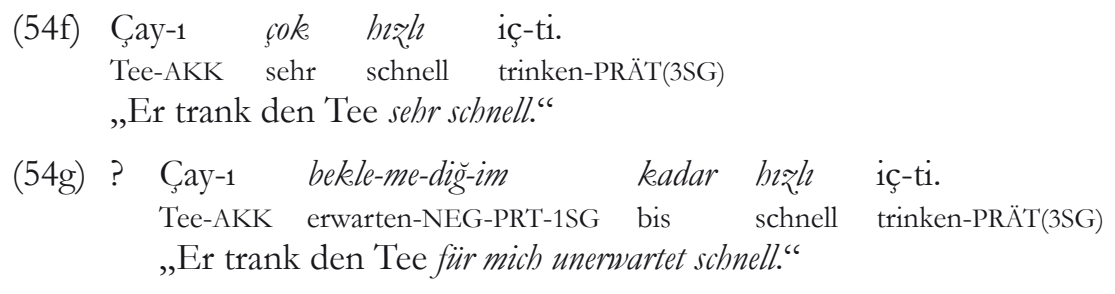

Die genannten Restriktionen bei den nichtabgeleiteten Adjektiven in der Funktion als Ereignismodifikatoren lassen die Vermutung zu, dass es sich hier nicht um Adjunkte der Verbalphrase, sondern um verbinkorporierte Formen handelt, die keinen eigenen Phrasenstatus haben, sondern semantisch, pragmatisch und syntaktisch eine Einheit mit dem Verb bilden. Diese Einheit lässt sich am ehesten als Kompositum beschreiben, vergleichbar den Objektinkorporationen im Türkischen (vgl. Schroeder 1999, S. 76 ff.).

\section{Zwischenergebnis: Form und Orientierung bei den ereignisinternen Adjunkten im Vergleich Deutsch-Türkisch}

Wenn wir nun Form und Orientierung bei den ereignisinternen Adjunkten im Deutschen und Türkischen gemäß den in der Einleitung zu Abschnitt 2 formulierten Ausgangsfragen aufeinander beziehen, erscheint das Deutsche stärker unterspezifiziert, während das Türkische stärker spezifiziert ist. Das Türkische weist bei den ereignisinternen Adjunkten unterschiedliche, produktiv gebildete Formen auf, die in Bezug auf ihre semantische Orientierung festgelegt sind, auch gibt es eine stärkere Tendenz zum geschlossenen Bezug bei den referentenorienierten ereignisinternen Adjunkten. Dies schließt Überlappungen der Funktion nicht aus, und insofern gibt es auch im Türkischen Vagheit - aber dann eben innerhalb eines festgelegten formalen Rahmens. Im Deutschen auf der anderen Seite finden wir eine starke Tendenz zur lexikalischen Festlegung der Orientierung - und das bedeutet, dass die prinzipielle Vagheit der Orientierung bei den ereignisinternen Adjunkten im Deutschen wesentlich stärker ist als im Türkischen.

Gleichzeitig sind vergleichbare Formen in den beiden Sprachen in ihrem grammatischen Status unterschiedlich einzuordnen: Nichtabgeleitete Adjektive sind im Deutschen grammatisch unbeschränkter, und sie sind die präferierte Form von ereignisinternen Adjunkten, während die gleiche Form im Türkischen syntaktisch und pragmatisch stark eingeschränkt ist. Umgekehrt sind die Explizitphrasen im Deutschen eine nicht grammatisch, son- 
dern stilistisch motivierte Form, während die morphologisch und lexikalisch ähnlich erscheinenden Explizitphrasen im Türkischen grammatisch motiviert sind.

Und zum dritten beobachten wir im Türkischen eine stärkere Tendenz zur Anbindung der ereignisinternen Adjunkte als im Deutschen. Dies geschieht zum einen über Possessivkongruenz mit dem Bezugsreferenten, nämlich bei den mit Instrumentalkasus gebildeten referentenorientierten Adjunkten, siehe Abschnitt 2.2.6 sowie 2.2.7. Und es geschieht bei den reduzierten (Konverb-)Sätzen über Koreferenz des Subjekts des Konverbs mit dem Ausdruck eines Ereignisbeteiligten (vgl. 2.2.9). Das gibt es auch im Deutschen, nämlich in Form der Partizipien, jedoch nicht in der formalen Vielfalt und der Frequenz, in der die Konverben im Türkischen eingesetzt werden. Es sei nochmals darauf hingewiesen, dass die wohl häufigste Form der ereignisinternen Adjunkte im Türkischen, nämlich die olarak-Phrase (siehe 2.2.1), ebenfalls auf eine Konverbform zurückzuführen ist.

\section{Typologische Korrelate}

Wäre dies eine rein kontrastive Arbeit, so könnte die Betrachtung der ereignisinternen Adjunkte im Deutschen und Türkischen hier abgeschlossen werden: Wir haben eingangs ein Tertium Comparationis etabliert (Abschnitt 1), haben dieses auf das Sprachenpaar Deutsch-Türkisch angewandt (Abschnitt 2) und Unterschiede und Gemeinsamkeiten formuliert (Abschnitt 3). Im Titel ist jedoch eine kontrastiv-typologische Untersuchung angekündigt, und das bedeutet, dass wir im Sinne des Eingangsstatements die Aufgabe haben, die festgestellten Unterschiede und Gemeinsamkeiten im System der betreffenden Sprachen zu verorten und dabei Parameter - „typologische Korrelate“ zu ermitteln, die sinnvoll mit den festgestellten Unterschieden korrelieren. Dies sollte zu einer Typenbildung in Form einer Hypothese führen. Letztere muss dann auf einen breiteren typologischen Vergleich angewendet und dergestalt getestet werden - aber das kann hier nicht geleistet werden, sondern ist Aufgabe einer breiteren typologischen Untersuchung auf der Basis einer sinnvollen Sprachenauswahl.

Zwei Korrelate - eines morphosyntaktisch, das andere lexikalisch-semantisch, mit starkem Bezug auf morphosyntaktische Kategorien - drängen sich auf.

\subsection{Ein morphosyntaktisches Korrelat: Konstituenten der Nominalphrase versus Konstituenten des Satzes}

Das erste Korrelat eignet sich dazu, die hier festgestellten Unterschiede zwischen Deutsch und Türkisch in einen weiteren formalen Zusammenhang zu verorten, nämlich den der morphologischen Unterscheidung bzw. 
Nicht-Unterscheidung zwischen Adjunkten in der Nominalphrase auf der einen und Adjunkten in der Verbalphrase und im Satz auf der anderen Seite. Boeder/Schroeder $(1998,2000)$ sowie Schroeder (2000) zeigen, dass das Türkische - und auch das Georgische - eine sehr starke Tendenz haben, formal zwischen Adjunkten in der Nominalphrase ( Attributen) und Adjunkten im Satz ( Adverbialen) zu unterscheiden. Als Attribute im Türkischen sind Adjektive (abgeleitete und unabgeleitete), Partizipialphrasen ( $\sim$ Relativsätze), Nominalphrasen im Genitiv sowie mit -ki gebildete nichtverbale attributive Sätze zulässig. Als Adverbiale sind Adverbien (abgeleitete und unabgeleitete), Konverbsätze ( adverbiale Sätze), Nominalphrasen mit adverbialem Kasus (Lokativ-, Ablativ- und Instrumentalkasus) sowie Postpositionalphrasen zulässig. Was als Attribut in der Nominalphrase fungieren kann, kann nicht in der gleichen Form Adverbial im Satz sein, sondern muss hierfür in adverbiale Form gebracht werden. Umgekehrt kann, was als Adverbial im Satz fungieren kann, nicht in der gleichen Form Attribut sein, sondern muss hierfür in Attributsform gebracht werden.

Das Deutsche dagegen erlaubt einen verhältnismäßig hohen Grad an Durchlässigkeit zwischen Nominalphrase und Satz, indem Adjektive, Adverbien und Präpositionalphrasen ohne formale Unterscheidung adverbial und attributiv verwendet werden können - die Adverbien und Präpositionalphrasen als post- und die Adjektive als präpositionale Attribute. Natürlich gibt es Beschränkungen, die uns letztendlich zu dem Schluss kommen lassen können, dass die unmarkierte Funktion von Adjektiven im Deutschen die der Attribute ist, und die unmarkierte Funktion von Adverbien und Präpositionalphrasen die der Satzadjunkte, dies ändert aber nichts an der prinzipiellen formalen Durchlässigkeit zwischen Adjunkten in der Nominalphrase und Adjunkten im Satz, die das Deutsche wesentlich vom Türkischen unterscheidet. ${ }^{20}$

In diesem Sinne wird das, was wir hier an spezifischen Unterschieden zwischen Deutsch und Türkisch auf der Ebene der ereignisinternen Adjunkte festgestellt haben, zum Teil einer Tendenz, die weitere Grammatikbereiche umfasst. Damit ist der Formenreichtum der ereignisinternen Adjunkte im Türkischen nicht erklärt, aber der in den beiden Sprachen unterschiedliche grammatische Status der unabgeleiteten Adjektive und der Explizitphrasen (siehe 2.3) wird verständlicher.

\subsection{Ein lexikalisch-semantisches Korrelat}

Das zweite Korrelat ist möglicherweise dazu geeignet, die geringere morphologische Spezifizierung bei den ereignisinternen Adjunkten im Deutschen gegenüber der höheren Spezifizierung bei diesen Formen im Türkischen zu erklären.

20 Siehe hierzu genauer Gunkel/Schlotthauer (in diesem Band). 
Bittet man einen türkischen Muttersprachler um eine semantisch adäquate Übersetzung von (77), so ist die Antwort (78):

(77) Er hüpfte ins Haus hinein.
sek-e sek-e
ev-e
gir-di.
hüpfen-REDUPL.KONV Haus-DAT sich.hineinbegeben-PRÄT(3SG)
„Er hüpfte ins Haus hinein.“ (wörtlich aber eher: „Er begab sich hüp- fend ins Haus hinein.")

In dem deutschen Beispiel (77) besteht der Stamm des Verbs, das die Prädikation trägt, aus zwei semantischen Komponenten, nämlich i) der Tatsache der Bewegung selbst und ii) dem Ausdruck einer bestimmten Art der Bewegung, die sich von anderen Arten der Bewegung unterscheidet - büpfen und nicht gehen, rennen, stolpern, torkeln etc. In dem türkischen Beispiel (78) besteht das zentrale Verb, das die Prädikation trägt, ebenfalls aus zwei semantischen Komponenten, nämlich i) der Tatsache der Bewegung selbst und ii) dem Ausdruck einer bestimmten Gerichtetheit der Bewegung, nämlich in einen geschlossenen Raum hinein.

Die semantischen Informationen, die im Bewegungsverb enthalten sind, unterscheiden sich also in den beiden Sprachen: Das deutsche Verb verbindet BEWEGUNG und ART UND WEISE der Bewegung miteinander; es ist ein „deskriptives Verb“ im Sinne Snell-Hornbys (1983). ${ }^{21}$ Die Information, dass es sich bei der Bewegung um eine gerichtete Bewegung handelt, wird nicht im Verbstamm kodiert, sondern in der Partikel binein, die Teil des Prädikatskomplexes ist. Sie drückt den WEG aus. Das Ziel der Bewegung (ins Haus) wird in einer direktionalen Ergänzung ausgedrückt. Das türkische Verb hingegen lexikalisiert die Tatsache der Bewegung und die Gerichtetheit der Bewegung, nämlich hier in einen geschlossenen Raum hinein. Es ist ein „deiktisches Verb“. Die ART UND WEISE der Bewegung wird außerhalb des Prädikatskomplexes kodiert, und zwar als Verbalphrasenadjunkt. Schematisch lässt sich dies folgendermaßen zusammenfassen:

\footnotetext{
21 Wobei „Art und Weise“ hier nicht lediglich als Art und Weise der Bewegung zu fassen ist. Snell-Hornby definiert deskriptive Verben wesentlich weiter als ,a semantic complex divisible into a core of verbal action (act-nucleus) and a descriptive, modifying complex of elements (modificant) expressed in the verb's basic definition by one or more adjectives or manner adverbs. The modificant may express modality of action (direct descriptivity), it may characterize one or more of the participants or it may specify circumstances surrounding the action (indirect descriptivity), or it may combine all three perspectives" (Snell-Hornby 1983, S. 43). So beinhaltet beispielsweise das Verb stolizieren als Handlungsnukleus vereinfacht „Fortbewegung/gehen/Agens +hum“, und als Modifikant sowohl die „direkt deskriptive“ Komponente der „Art und Weise“ der Bewegung (steif gehen) als auch eine referentenbezogene „indirekt deskriptive“ Komponente (steif und hochmütig).
} 


\section{Deutsch}

\begin{tabular}{|l|l|l|}
\hline BEWEGUNG \& ART UND WEISE & QUELLE/ZIEL & WEG \\
\hline finites Verb & Adpositionalphrase & Satellit \\
\hline büpfte & ins Haus & hinein \\
\hline
\end{tabular}

Türkisch

\begin{tabular}{|l|l|l|}
\hline ART UND WEISE & QUELLE/ZIEL & BEWEGUNG \& WEG \\
\hline Verbalphrasenadjunkt & $\begin{array}{l}\text { Adpositionalphrase/ } \\
\text { adverbialer Kasus }\end{array}$ & finites Verb \\
\hline seke seke & eve & girdi \\
\hline
\end{tabular}

Tab. 1: Die Komponenten von Bewegungsereignissen

Diese Opposition korreliert mit folgenden, vor allem auf Slobin (2000, 2004) und Özçalıskan/Slobin $(2000,2003)$ gestützten Beobachtungen in Bezug auf Tendenzen bei den Distributionen von Verbklassen im Türkischen, denen ich das Deutsche gegenüberstelle:

- Im Türkischen werden Sätze, die eine gerichtete Bewegung ausdrücken, in der Regel mit deiktischen (Bewegungs-)Verben gebildet, die eine Direktionalergänzung erfordern. Die deiktischen Verben drücken eine spezifische Form der Gerichtetheit aus (u.A. gir- „sich hineinbegeben“, glk„sich hinausbegeben“, in- „sich abwärts begeben“, gel- „[von QUELLE] kommen“, git- „sich [auf ZIEL] hin bewegen“). Sie haben jedoch keine deskriptive Komponente, d.h. sie geben keinerlei Information über die Art und Weise der Bewegung.

- Das Deutsche hat keine entsprechend geschlossene Gruppe von deiktischen Verben. Allerdings finden die polysemen Verben kommen und gehen auch als deiktische Verben Verwendung (vgl. Di Meola 1994).

- Deskriptive Verben im Deutschen und Türkischen unterscheiden sich stark bezüglich Transitivität und Erweiterungsmöglichkeiten. Türkische deskriptive Verben sind in der Regel intransitiv und nicht durch Wegund Direktionalergänzungen erweiterbar. Deutsche deskriptive Verben können transitiv und intransitiv sein und sie sind durch Weg- und Direktionalergänzungen erweiterbar. Zum Beispiel ist, wie wir in Beispiel (77) gesehen haben, das deutsche deskriptive Verb büpfen problemlos als finites Verb mit einer Direktionalangabe kombinierbar; sein türkisches Äquivalent sek- in (78) dagegen ist nicht erweiterbar:

(78a) *Ev-e sek-ti.

Haus-DAT hüpfen-PRÄT(3SG) 
- Deskriptive Bewegungsverben im Türkischen finden sich eher in der Form von Konverben ( Gerundien) als in der Form finiter Prädikate. Das heißt, sie dienen vorrangig als Modifikatoren im Rahmen eines gerichteten Bewegungsereignisses, das von einem deiktischen Verb als finitem Prädikat ausgedrückt ist.

Insgesamt verfügt das Deutsche zudem über mehr deskriptive Verben als das Türkische und über einen wesentlich höheren semantischen Differenzierungsgrad bei diesen Verben. ${ }^{22}$

In der Typologie Talmys $(1991,2000)$ wird Deutsch mit diesen (und weiteren) Eigenschaften zu einer satellite framed language gezählt; Türkisch dagegen ist eine verb framed language. Der für Talmy entscheidende Punkt für diese Klassifikation ist dabei, dass bei satellite framed-Sprachen der raumbezogene „Rahmen“ ( frame) des Bewegungsereignisses außerhalb des lexikalischen Stammes des die Prädikation tragenden Verbs ausgedrückt wird, nämlich in einem „Satelliten“ zum Verb, während bei den verb framed Sprachen der raumbezogene Rahmen im Verbstamm selbst ausgedrückt wird. Für meine Argumentation ist jedoch das Korrelat dieser Differenzierung entscheidender, nämlich dass satellite framed-Sprachen allgemein eine stärkere Tendenz haben, die ART UND WEISE der Bewegung im zentralen Verb des Satzes zu lexikalisieren, während verb framed-Sprachen eher dazu tendieren, die Kodierung der ART UND WEISE außerhalb des zentralen Verbs zu realisieren. Weitere Sprachen, die sich in diesem Zusammenhang dem Deutschen ähnlich verhalten, sind das Englische und andere germanische Sprachen; ähnlich wie das Türkische verhalten sich u.a. Spanisch, Französisch, Japanisch und Koreanisch.

Die unterschiedlichen Distributionen der beiden Sprachen Deutsch und Türkisch in Bezug auf die typologische Differenzierung zwischen satelliteund verb framed-Sprachen lässt sich sinnvoll mit der geringeren morphologischen Spezifizierung bei den ereignisinternen Adjunkten im Deutschen gegenüber der höheren Spezifizierung bei diesen Formen im Türkischen korrelieren, und zwar mit dem Argument, dass auf den ereignisinternen Adjunkten im Türkischen ein größeres funktionales Gewicht als auf den ereignisinternen Adjunkten im Deutschen liegt - die ereignisinternen Adjunkte nämlich sind es, die das funktionale Gewicht des Ausdrucks der ART UND WEISE der Bewegung zu tragen haben. Dieses Argument setzt natürlich ein funktionales Sprachverständnis voraus, das davon ausgeht, dass sich eine Differenzierung von Formen proportional zur Bedeutung einer Kategorie für die Repräsentation von Erfahrung verhält, und dass Ereignismodifikation - wie immer sie auch ausgedrückt wird - eine bedeutende Kategorie ist. ${ }^{23}$

\footnotetext{
22 Siehe die beeindruckende Analyse von Snell-Hornby (1983), die Deutsch und Englisch in Bezug auf verbale Deskriptivität kontrastiert.

23 Weiter zu überlegen wäre, ob die satellite-versus verb framed-Unterscheidung nicht auch in eine kontrastive Untersuchung der Konstituierung von Ereignissen im Sinne der Dik'schen
} 


\section{Kommentar zum Abschluss}

Die wesentlichen Ergebnisse des kontrastiven Teils dieser Untersuchung sind in Abschnitt 3 bereits formuliert worden und brauchen nicht wiederholt zu werden. Die in 4 entwickelten Korrelate bilden Hypothesen von „sinnvollen Zusammenhängen“, mehr nicht: Wir stoßen an die Grenzen des kontrastiven Vergleichs zweier Sprachen. Ohne den „feinkörnigen Vergleich“ aber wäre die Hypothesenbildung nicht möglich gewesen. Der kontrastive Vergleich zeigt hier seine Stärke als Partner einer holistisch orientierten Typologie im Sinne der in der Einleitung formulierten Charakterisierung.

\section{Abkürzungen in den Interlinearisierungen}

$\begin{array}{llll}\text { ABL } & \text { Ablativ } & \text { IMPOSS } & \text { Impossibilis } \\ \text { ADJ } & \text { Adjektiv } & \text { INF } & \text { Infinitiv } \\ \text { ADV } & \text { Adverb } & \text { INSTR } & \text { Instrumental } \\ \text { AKK } & \text { Akkusativ } & \text { INTENS } & \text { Intensivierung } \\ \text { AOR } & \text { Aorist } & \text { JUNK } & \text { Junktor } \\ \text { ATTR } & \text { Attribut } & \text { KAUS } & \text { Kausativ } \\ \text { DAT } & \text { Dativ } & \text { KOMP } & \text { Komparativ } \\ \text { DEPIKT } & \text { Depiktiv } & \text { KONJ } & \text { Konjunktiv } \\ \text { EVID } & \text { Evidentialis } & \text { KONV } & \text { Konverb } \\ \text { FAKT } & \text { Faktiv } & \text { KOP } & \text { Kopula } \\ \text { FUT } & \text { Futur } & \text { LOK } & \text { Lokativ } \\ \text { GEN } & \text { Genitiv } & \text { NEG } & \text { Negativ } \\ \text { HABIT } & \text { Habitualis } & \text { NOM } & \text { Nominalisierung } \\ \text { IDEO } & \text { Ideophon } & \text { OBLIG } & \text { Obligativ } \\ \text { IMP } & \text { Imperativ } & \text { PART } & \text { Partikel }\end{array}$

(1975) Ereignistypologie eingebettet werden könnte. Das Deutsche scheint unterschiedliche Ereignistypen stärker lexikalisch am Verb zu unterscheiden (z.B. sitzen - Position vs. setzen Aktivität), während das Türkische stärker die Modifikation durch Adjunkte funktionalisiert, und hierfür auch die ereignisinternen Adjunkte heranzieht, vgl. sessiz sessiz otur- (still (REDUPL) sitzen) ,still sitzen“: Die Position sitzen wird als Vorgang modifiziert; vgl. dagegen sessiz-ce otur- (still-ADV sitzen) ,,(sich) still setzen“: Die Aktivität wird in ihrer Ausführung modifiziert. Das Verb otur- selbst unterscheidet nicht zwischen den Ereignistypen. In Ermangelung systematischer vergleichender Untersuchungen zur Ereignistypologie - Sasses (Hg.) (1991) diesbezüglicher Ansatz wurde leider nicht fortgeführt - müssen wir dies allerdings zurückstellen. 


$\begin{array}{llll}\text { PASS } & \text { Passiv } & \text { QUE } & \text { Interrogativ } \\ \text { PERF } & \text { Perfekt } & \text { REDUPL } & \text { Reduplikation } \\ \text { PL } & \text { Plural } & \text { RESULT } & \text { Resultativ } \\ \text { POSS } & \text { Possessiv } & \text { S } & \text { Subjekt } \\ \text { POT } & \text { Potentialis } & \text { SG } & \text { Singular } \\ \text { PRÄS } & \text { Präsens } & \text { SUPER } & \text { Superlativ } \\ \text { PRÄT } & \text { Präteritum } & 1 & \text { 1. Person } \\ \text { PROGR } & \text { Progressiv } & 2 & \text { 2. Person } \\ \text { PRT } & \text { Partizip } & 3 & \text { 3. Person }\end{array}$

\section{Literatur}

Aarts, Bas (1992): Small clauses in English. The nonverbal type. (= Topics in English Linguistics 8 ). Berlin.

Aarts, Bas (1995): Secondary predicates in English. In: Aarts, Bas/Meyer, Charles F. (Hg.) (1995): The verb in contemporary English: theory and description. Cambridge, S. 75-101.

Abraham, Werner (1995): Deutsche Syntax im Sprachenvergleich. Grundlegung einer typologischen Syntax des Deutschen. (= Studien zur deutschen Grammatik 41). Tübingen.

Bartsch, Renate (1976): The grammar of adverbials. A study in the semantics and syntax of adverbial constructions. (= North-Holland Linguistic Series 16). Amsterdam.

Bausewein, Karin (1990): Akkusativobjekt, Akkusativobjektsätze und Objektsprädikate im Deutschen. Untersuchungen zu ihrer Syntax und Semantik. (= Linguistische Arbeiten 251). Tübingen.

Bellert, Irena (1977): On semantic and distributional properties of sentential adverbs. In: Linguistic Inquiry 8, S. 337-350.

Boeder, Winfried/Schroeder, Christoph (1998): Attribution und sekundäre Prädikate im Sprachvergleich: Deutsch, Englisch, Kurdisch, Georgisch, Türkisch. In: Sprachtypologie und Universalienforschung 51, S. 207-227.

Boeder, Winfried/Schroeder, Christoph (2000): Relational coding in Georgian and Turkish noun phrases: syntax, derivational morphology, and „linking“ by means of participles. In: Turkic Languages 4, S. 153-204.

Buysschaert, Joost (1982): Criteria for the classification of English adverbials. (= Varhandelingen van de Koninklijke Academie voor Wetenschapen, Letteren en Schone Kunsten van Belgie, Klasse der Letteren, Jaargang 44, Nr. 99). Brüssel.

Chomsky, Noam (1981): Lectures on government and binding. (= Studies in Generative Grammar 9). Dordrecht u.a. 
Di Meola, Claudio (1994). Kommen und gehen. Eine kognitiv-linguistische Untersuchung der Polysemie deiktischer Bewegungsverben. (= Linguistische Arbeiten 325). Tübingen.

Dik, Simon (1975): The semantic representation of manner adverbials. In: Kraak, Albert (Hg.): Linguistics in the Netherlands 1972-1973. Assen, S. 96-121.

Dik, Simon/Hengeveld, Kees/Vester, Elseline/Vet, Co (1990): The hierarchical structure of the clause and the typology of adverbial satellites. In: Nuyts, Jan/Bolkestein, A. Machtelt/Vet, Co (Hg.): Layers and levels of representation in language theory: a functional view. (= Pragmatics \& Beyond, New Series 13). Amsterdam/Philadelphia, S. 25-70.

Dowty, David (1972): Temporally restricted adjectives. In: Syntax and Semantics 1, S. $51-62$.

Eisenberg, Peter (1999): Grundriß der deutschen Grammatik. Bd. 2: Der Satz. Stuttgart u.a.

Engel, Ulrich (1988): Deutsche Grammatik. Heidelberg.

Foley, William/Van Valin Jr., Robert D. (1984): Functional syntax and universal grammar. (= Cambridge Studies in Linguistics 38). Cambridge.

Frey, Werner/Pittner, Karin (1998): Zur Positionierung der Adverbiale im Mittelfeld. In: Linguistische Berichte 176, S. 489-534.

Geuder, Wilhelm (2000): Oriented adverbs. Issues in the lexical semantics of event adverbs. Diss. Tübingen.

Geuder, Wilhelm (2004): Depictives and transparent adverbs. In: Austin, Jennifer/ Rauh, Gisa/Engelberg, Stefan (Hg.) (2004). Adverbials. The interplay between meaning, context and syntactic structure. (= Linguistik aktuell 70). Amsterdam, S. 131-166.

Fabricius-Hansen, Cathrine/Ramm, Wiebke (i.d.Bd.): Ein Parallelkorpus im Einsatz: grammatische Variation im Bereich der Satzverbindung und Informationsverteilung (Deutsch - Norwegisch - Englisch/Französisch).

Göksel, Aslı/Kerslake, Celia (Hg.) (2000): Studies on Turkish and Turkic Languages. Proceedings of the Ninth International Conference on Turkish Linguistics, Lincoln College, Oxford, August 12-14, 1998. (= Turcologica 46). Wiesbaden.

Gunkel, Lutz/Schlotthauer, Susan (i.d.Bd.): Adnominale Adverbien im europäischen Vergleich.

Helbig, Gerhard/Buscha, Joachim (1989): Deutsche Grammatik. Ein Handbuch für den Ausländerunterricht. 12., unveränd. Aufl. Leipzig.

Jackendoff, Ray S. (1972): Semantic interpretation in generative grammar. Cambridge, MA.

Jendraschek, Gerd (2001): Semantic and structural properties of Turkish ideophones. In: Turkic Languages 5, S. 88-103.

König, Ekkehard (i.d.Bd.): Zur Standortbestimmung der Kontrastiven Linguistik innerhalb der vergleichenden Sprachwissenschaft. 
Lehmann, Christian (1988): Towards a typology of clause linkage. In: Haiman, John/ Thompson, Sandra A. (Hg.): Clause combining in grammar and discourse. (= Typological Studies in Language 18). Amsterdam/Philadelphia, S. 181-225.

Maas, Utz (2000): Überlegungen zu einer typologisch orientierten kontrastiven Sprachwissenschaft, am Beispiel Arabisch - Deutsch. In: Maas, Utz/Selmy, El-Sayed/ Ahmed, Mostafa (Hg.): Perspektiven eines typologisch orientierten Sprachvergleichs Deutsch - Arabisch, Arabisch - Deutsch. Kairo, S. 1-14.

Maienborn, Claudia/Schäfer, Martin (ersch.demn.): Adverbs and adverbials. In: Maienborn, Claudia/Heusinger, Klaus von/Portner, Paul (Hg.): Semantics. An international handbook of natural language meaning. Bd. 1. (= Handbücher zur Sprachund Kommunikationswissenschaft 33.1). Berlin/New York.

Müller, Stefan (2003): Complex predicates. Verbal complexes, resultative constructions, and particle verbs in German. (= Studies in Constraint-Based Lexicalism 13). Stanford.

Müller-Bardey, Thomas (1990): Koprädikation als grundlegende syntaktische Operation. In: Seiler, Hansjakob et al. (Hg.): Internationales Interdisziplinäres Kolloquium ,Sprache und Denken: Variation und Invarianz in Linguistik und Nachbardisziplinen“. Bd. II. (= Arbeiten des Kölner Universalien-Projekts 80). Köln, S. 1-20.

Napoli, Donna Jo (1989): Predication theory. A case study for indexing theory. (= Cambridge Studies in Linguistics 50). Cambridge.

Nichols, Johanna (1978): Secondary predicates. In: Berkeley Linguistics Society (BLS) 4, S. $114-127$.

Nikula, Henrik (1982): „Temporalisierte“ Adjektive. Über Linearität, Temporalität und Kausalität in der Sprache. In: Studia Neophilologica 54, S. 81-98.

Özçalışkan, Şeyda/Slobin, Dan (2000): Manner of movement in monolingual and bilingual adult narratives: Turkish vs. English. In: Göksel/Kerslake (Hg.), S. 253-262.

Özçalıskan, Şeyda/Slobin, Dan (2003): Codability effects on the expression of manner of motion in Turkish and English. In: Özsoy, Sumru et al. (Hg.): Studies in Turkish linguistics. Istanbul, S. 259-270.

Pinkster, Harm (1990): Latin syntax and semantics. London.

Pittner, Karin (1999): Adverbiale im Deutschen. Untersuchungen zu ihrer Stellung und Interpretation. (= Studien zur deutschen Grammatik 60). Tübingen.

Pittner, Karin (2002): Adverbien der Art und Weise im Deutschen und Englischen: zu ihrer Stellung und Interpretation. In: Schmöe, Friederike (Hg.): Das Adverb. Zentrum und Peripherie einer Wortklasse. Wien, S. 43-60.

Plank, Frans (1985): Prädikativ und Koprädikativ. In: Zeitschrift für Germanistische Linguistik (ZGL) 13, S. 154-185.

Platt, John T./Platt, Heidi K. (1972): Orientation of manner adverbials. In: Papers in Linguistics 5, S. 227-249.

Quirk, Randolph et al. (1985): A comprehensive grammar of the English language. London. 
Ramat, Paola/Ricca, Davide (1998): Sentence adverbs in the languages of Europe. In: van der Auwera, Johan (Hg.) (in collaboration with Baoill, Dónall P.Ó.): Adverbial constructions in the languages of Europe. (= Empirical Approaches to Language Typology/EUROTYP 20-3). Berlin/New York, S. 187-275.

Rath, Rainer (1972): Adverbialisierte Adjektive im Deutschen. In: Linguistische Berichte 20, S. $1-18$.

Renz, Ingrid (1993): Adverbiale im Deutschen. Ein Vorschlag zu ihrer Klassifikation und unifikationsbasierten Interpretation. (= Linguistische Arbeiten 298). Tübingen.

Renz, Matthias/Hentschel, Gerd (2011): On adjectives and adverbs expressing ,nakedness $^{6}$ and ,barefootedness' in Polish and Russian: a study on morphosyntactic variation. In: Russian Linguistics 35, S. 63-87.

Rijkhoff, Jan (2004): The noun phrase. Oxford u.a.

Sasse, Hans-Jürgen (Hg.) (1991): Aspektsysteme. (= Arbeitspapier, Institut für Sprachwissenschaft, Universität zu Köln, NF 14). Köln.

van Schaaik, Gerjan (Hg.) (1990-2000): Turkish Text Corpus. Leiden.

Schroeder, Christoph (1999): The Turkish nominal phrase in spoken discourse. (= Turcologica 40). Wiesbaden.

Schroeder, Christoph (2000): Attribution in Turkish and the function of -ki. In: Göksel/Kerslake (Hg.), S. 205-216.

Schroeder, Christoph (2004): Depiktive im Sprachvergleich Deutsch-Türkisch. Eine kontrastiv-typologische Analyse. Habilitationsschrift (unveröff.). Osnabrück.

Schroeder, Christoph (2008): Adverbial modification and secondary predicates in Turkish: a typological perspective. In: Schroeder, Christoph/Hentschel, Gerd/Boeder, Winfried (Hg.): Secondary predicates in Eastern European languages and beyond. Oldenburg, S. 339-358.

Schultze-Berndt, Eva/Himmelmann, Nikolaus P. (2004): Depictive secondary predicates in crosslinguistic perspective. In: Linguistic Typology 8, 1, S. 59-131.

Slobin, Dan I. (2000): Verbalized events: A dynamic approach to linguistic relativity and determinism. In: Niemeier, Susanne/Dirven, René (Hg.): Evidence for linguistic relativity. Amsterdam/Philadelphia, S. 107-138.

Slobin, Dan I. (2004): The many ways to search for a frog: linguistic typology and the expression of motion events. In: Strömqvist, Sven/Verhoeven, Ludo (Hg.): Relating events in narrative: Typological and contextual perspectives. Bd. 2. Mahwah, NJ, S. 219-257.

Snell-Hornby, Mary (1983): Verb descriptivity in German and English. A contrastive study in semantic fields. (= Anglistische Forschungen 158). Heidelberg.

Staudinger, Bernhard (1997): Sätzchen: Small Clauses im Deutschen. (= Linguistische Arbeiten 363). Tübingen.

Steube, Anita (1994): Syntaktische und semantische Eigenschaften sekundärer Prädikate. In: Steube, Anita/Zybatow, Gerhild (Hg.): Zur Satzwertigkeit von Infinitiven und Small clauses. (= Linguistische Arbeiten 315). Tübingen, S. 243-264. 
Stowell, Timothy (1983): Subjects across categories. In: The Linguistic Review 2, S. $285-$ 312.

Talmy, Leonard (1991): Path to realization: a typology of event conflation. In: Berkeley Linguistics Society (BLS) 17, S. 480-519.

Talmy, Leonard (2000): Toward a cognitive semantics. Bd. II: Typology and process in concept structuring. Cambridge, MA.

Tenny, Carol L. (2000): Core events and adverbial modification. In: Tenny, Carol/Pustejovsky, James (Hg.): Events as grammatical objects: the converging perspectives of lexical semantics and syntax. (= CSLI Lecture Notes 100). Stanford, S. 285-334.

Van Valin, Robert D./LaPolla, Randy J. (1997): Syntax. Structure, meaning and function. Cambridge u.a.

Zifonun, Gisela/Hoffmann, Ludger/Strecker, Bruno (1997): Grammatik der deutschen Sprache. Bd. 2. (= Schriften des Instituts für Deutsche Sprache 7.2). Berlin/New York. 
